\title{
Antiphospholipid antibodies and Mycoplasma pneumoniae infection
}

\author{
Neil Snowden, Philip B. Wilson, Maurice Longson ${ }^{1 *}$ and Richard S.H. \\ Pumphrey
}

Regional Immunology Service, St Mary's Hospital Manchester, M130JH and ${ }^{1}$ The Virology Unit, Booth Hall Children's Hospital, Manchester, UK.

\begin{abstract}
Summary: Anticardiolipin antibody levels were measured in 57 patients with Mycoplasma pneumoniae infection and 21 patients with other infections. Significantly more patients in the mycoplamsa group had increased IgM and IgG anticardiolipin. Within the mycoplasma group significantly higher titres were found in patients with severe infection (assessed by need for hospital admission) and in patients with cold agglutinins. A tendency for particularly high titres to occur in patients with extra-pulmonary complications was identified.
\end{abstract}

\section{Introduction}

Antibodies to negatively charged phospholipids, such as cardiolipin, are associated with nonvasculitic thrombosis, recurrent spontaneous abortion, thrombocytopenia and neurological disease. $^{1,2}$ This association was first noted in systemic lupus erythematosus (SLE) where antiphospholipid antibodies (aPL) are very closely linked with ${ }^{3}$ (but are not identical to ${ }^{4}$ ) the so-called 'lupus anticoagulant'. Recently, patients have been described with high titre aPL, thrombosis and recurrent abortion but with no evidence of connective tissue disease or other immunopathology. These patients may constitute a 'primary antiphospholipid antibody syndrome'. 5,6 Low titre aPL have been demonstrated in a number of other conditions: acute infections (infectious mononucleosis, ${ }^{7}$ human immunodeficiency virus infection ${ }^{8}$, malignancy, ischaemic heart disease ${ }^{9}$ and in the healthy elderly.$^{10}$ The significance of this is unclear; in general it is felt to be representative of the low grade autoimmune responses of little functional significance which accompany tissue damage and ageing ${ }^{11}$ (in contrast with the aPL responses in SLE and the primary aPL syndrome where there is some evidence that these antibodies play a direct role in the pathogenesis of the associated complications ${ }^{12}$ ).

During development of an enzyme-linked immunosorbent assay (ELISA) for aPL (using cardiolipin as antigen) at the North West Regional

Correspondence: N. Snowden M.R.C.P.

*Present address: Faculty of Medicine and Health Sciences, Al Ain, United Arab Emirates.

Accepted: 4 January 1990
Immunology Service, large numbers of serum samples from normal controls and patients were analysed. In addition to the expected positive results from patients with SLE and low titre positives from other conditions, it was noted that in a few patients, markedly increased titres of aPL occurred unexpectedly. Particularly high titres were noted in the serum of one patient with Mycoplasma pneumoniae infection. This was interesting in view of the recognized extra pulmonary complications of mycoplasma infection, such as haemolysis, neurological disease ${ }^{13}$ and stroke, ${ }^{14,15}$ some of which may have an immunological basis.

We therefore examined the hypothesis that patients with Mycoplasma pneumoniae infection were particularly likely to develop high titres of aPL. A retrospective analysis of sera from patients with known $M$. pneumoniae infection was undertaken. We attempted to correlate aPL levels with severity of illness and complications.

We also had the opportunity to study serum from one patient with an ischaemic stroke in association with $M$. pneumoniae infection (not from the retrospective series).

\section{Materials and methods}

\section{Patients}

Details of the patient with stroke in association with Mycoplasma pneumoniae infection have been previously reported. ${ }^{14}$ Briefly, a 31 year old previously fit male presented with a 3-week history 
of malaise, dyspnoea and cough and a 1-day history of drowsiness and left sided weakness. Chest X-ray revealed left sided pneumonic changes, a computed tomographic (CT) scan of the head showed a right cerebral hemisphere infarct and serological testing revealed a four-fold increase in complement fixation titres to $M$. pneumoniae. No source for embolism or other risk factor for stroke was identified.

Serum samples were obtained from all patients with serological evidence of $M$. pneumoniae infection over a 2-year period (January 1986-January 1988) detected at the North Manchester Regional Virus Laboratory. Names of patients were obtained from the laboratory weekly returns to the Communicable Disease Surveillance Centre. All sera had been stored at $-40^{\circ} \mathrm{C}$ and aPL levels were measured within 48 hours of thawing. Where possible, paired serum samples were retrieved (one from the acute phase of the illness and the other taken 7-14 days later), but in most cases only the second (convalescent) sample was available. Serological evidence of $M$. pneumoniae infection was defined as a four-fold increase in complement fixation (CF) titre between the first and second samples, or a single titre greater than or equal to $1 / 320$ in the context of an illness consistent with $M$. pneumoniae infection. Sera were obtained from 57 patients in all ( 32 female, 25 male. Mean age 23. Range $2 \frac{1}{2}-58 ; 19$ patients aged 11 or under) with paired samples from 17. The CF assay was performed according to a standard protocol using a five $-0.2 \mathrm{ml}$ volume test.

Sera were also obtained from 21 patients (mean age 14 ; range $1-46$ ) with other infections presenting to the laboratory over the same time period (4 hepatitis A, 2 Chlamydia psittaci, 4 parvovirus, 1 Herpes simplex encephalitis, 1 Coxiella burnettii, 1 Varicella zoster, 1 cytomegalovirus, 6 rubella and 1 influenza B. Infection was defined in terms of four-fold rise in antibody titre, organism-specific IgM or virus isolation)

A letter requesting further clinical details was sent to each of the consultants and general practitioners supervising the cases of mycoplasma infection. Information was sought on: (1) the presenting illness (e.g. pneumonia, upper respiratory tract infection, (2) the severity of illness (e.g. treated at home, hospitalized, died), (3) acute extrapulmonary complications (e.g. haemolysis, neurological disease, skin rashes), (4) any illness (with particular reference to vascular and neurological disease and complication of pregnancy) occurring in the 3 months following infection, (5) the presence or absence of cold agglutinins. These details were either recorded on a standard proforma by the clinician or extracted from the case notes by one of the investigators, in each case without knowledge of the anticardiolipin titres.

\section{Anticardiolipin ELISA}

Antibodies to cardiolipin were detected by an isotype specific ELISA, after the method of Harris and co-workers. ${ }^{16,17}$

Cardiolipin (Sigma) was prepared to a concentration of $50 \mu \mathrm{g} / \mathrm{ml}$ in ethanol; $100 \mu \mathrm{l}$ of this solution was placed in each well of a 96 well polystyrene microtitre plate. The ethanol was then evaporated by overnight storage at $4^{\circ} \mathrm{C}$. The plates were blocked for 60 minutes using $10 \%$ newborn calf serum (Flow) in phosphate buffered saline (NCS/PBS). The blocking solution was then shaken from the plates. The serum samples were diluted $1 / 10$ in NCS/PBS and $100 \mu$ l were added to the wells in duplicate. One row (8 wells) was not exposed to serum: NCS/PBS only was added to these wells. Each plate incorporated serial doubling dilutions (from $1 / 10$ to $1 / 640$ ) of a standard serum from a patient with SLE and high titres of IgM and IgG anticardiolipin (aliquotted and stored at $-40^{\circ} \mathrm{C}$ ). This was used to derive a standard curve for each plate. Each plate also incorporated another known positive serum as an interplate quality control. Serum samples from 19 normal controls (mean age 23; range $2 \frac{1}{2}-58$ ) were also tested in order to provide further validation for the normal ranges used. The plates were incubated for 60 minutes at room temperature and then washed three times in PBS. One hundred $\mu$ l of peroxidase conjugated rabbit antibody to human IgG or IgM (Dako) diluted 1/500 in NCS/PBS was added to each well.

Following a further 1 hour incubation the plates were washed three times in PBS and $200 \mu \mathrm{l}$ of $1 \mathrm{mM}$-ABTS (Sigma A-1888) containing $0.1 \mathrm{mM}$ hydrogen peroxide in citrate phosphate buffer (pH 5.4) was added to each well. The reactions were then allowed to develop until the optical density of the $1 / 10$ dilution of the standard serum read approximately 1 . The plates were then read using a Titertek ${ }^{\circledR}$ Multiskan plate reader. The standard curve (optical density versus dilution) was plotted and optical densities from the test samples were read relative to this curve and expressed in arbitrary units derived from the mean and 3 standard deviations of a large number of healthy controls. For IgG anticardiolipin normal was defined as $\leqslant 10 \mathrm{U}$ and for IgM anticardiolipin, $\leqslant 15 \mathrm{U}$. The use of a standard curve reduces interplate error due to variables such as time and temperature. Plates yielding more than $10 \%$ variation from previous laboratory values for the quality control sample were discarded. Samples with anticardiolipin levels outside the most sensitive zone of the standard curve were retested at $1 / 100$ dilution. The 19 controls gave means $( \pm 3$ standard deviation), for IgM and IgG isotypes, of $3.3( \pm 10.2) \mathrm{U}$ and $2.8( \pm 5.7) \mathrm{U}$ respectively. 
The assay described was developed, and the units of measurement defined, before the report by Harris et al. ${ }^{17}$ of the first attempt at inter-laboratory standardization of the anticardiolipin assay. Subsequent calibration of our assay against 5 standard sera obtained from St Thomas' Hospital, London suggests that, for IgM, 1 'Manchester unit' is approximately equalivent to 0.6 'Harris units' and for IgG, 1 'Manchester unit' is approximately equivalent to 2.5 'Harris units'.

IgM rheumatoid factor was detected by the agglutination of dyed gel particles coated with rabbit IgG (RAPA, Sera-Diagnostics). Other autoantibodies were detected by indirect immunofluorescence using as substrate (a) composite tissue blocks of rat stomach, liver and kidney and (b) culture Hep 2 cells. Samples were screened at a 1/20 dilution in PBS and diluted as appropriate.

\section{Statistics}

Comparison between test and control group medians was made using the Mann Whitney U test. Comparisons between test and control groups and the laboratory normal range were made by dividing patients into 3 groups: those with aCL levels (a) within the normal range, (b) above normal and (c) above twice normal. The null hypothesis that the distribution of test and control patients within these groups was identical was tested using $\chi^{2}$ (or Fisher's exact test if numbers were small). Comparison between anticardiolipin results and other laboratory data was made using Spearman's rank order correlation coefficient. Two tailed tests were used in all cases.

\section{Results}

The patient with mycoplasma infection and a stroke had IgM and IgG anticardiolipin (aCL) levels of 46 and 7 respectively (sample taken 2 days after admission to hospital).

The anticardiolipin titres from the retrospective study are shown in Figure 1 (for patients with paired samples, only the values from the second sample are shown). The median absolute values were significantly higher in the mycoplasma groups (Mann-Whitney U test, $P<0.001$ for both IgM and IgG aCL). Significantly more patients in the mycoplasma group had IgM and IgG anticardiolipin titres outside the normal range and, for IgM aCL, greater than twice the normal range (Table I).

Analysis of the 17 paired samples is shown in Table II. Seven of the 8 samples positive for IgG anticardiolipin on both occasions, or becoming positive on the second sample had positive IgM $\mathrm{aCL}$. Four of 8 patients negative for IgG aCL on

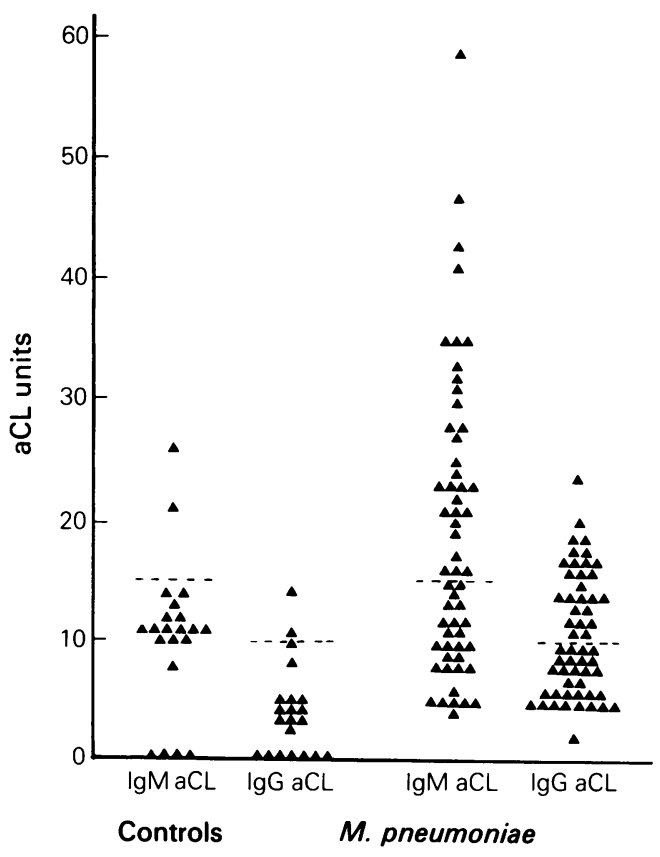

Figure 1 Levels of IgM and IgG anticardiolipin (aCL) in 57 patients with Mycoplasma pneumoniae infection and 21 patients with other infections. Hatched line indicates laboratory normal range.

Table I Number of patients with IgM and IgG anticardiolipin (aCL) greater than normal range and greater than twice normal range.

\begin{tabular}{lcccccccc}
\hline & \multicolumn{3}{c}{$I g M a C L / U$} & & \multicolumn{3}{c}{$\operatorname{Ig} a a C L / U$} \\
\cline { 2 - 3 } \cline { 6 - 8 } & $\leqslant 15$ & $15-30$ & $\geqslant 30$ & $\leqslant 10$ & $10-20$ & $\geqslant 20$ \\
\hline $\begin{array}{l}\text { Mycoplasma } \\
\text { pneumoniae } \\
(n=27)\end{array}$ & 27 & 19 & 11 & 30 & 24 & 3 \\
$\begin{array}{l}\text { Controls } \\
(n=21)\end{array}$ & 19 & 2 & 0 & 19 & 2 & 0 \\
& & & & & & & &
\end{tabular}

For IgM aCL $\chi^{2}=12.26$ ( 2 degrees of freedom) $P<0.01$. For IgG aCL $\chi^{2}=9.41, P<0.01$ (1 degree of freedom, comparing values greater and less than normal only. Numbers with values $>20$ too small for comparison by $\chi^{2}$.

both occasions had positive IgM. The median IgM and IgG aCL levels from the second of the paired samples were not significantly different from those from patients with only a single (convalescent) samples (Mann-Whitney U test $P>0.1$ ).

Clinical details were obtained on $40 / 57(70 \%)$ patients. Twenty seven patients were hospitalized and 13 were treated at home. Twenty six of the hospitalized patients had clinical and/or radiographic evidence of pneumonia. One developed bilateral facial nerve palsies (lower motor neu- 
Table II Paired serum samples: numbers of patients with acute (sample 1) and convalescent (sample 2) IgM and IgG aCL above and below the normal range.

\begin{tabular}{llccc}
\hline & $\begin{array}{l}\text { Both } \\
\text { positive }\end{array}$ & $\begin{array}{l}\text { Sample 1 negative } \\
\text { Sample 2 positive }\end{array}$ & $\begin{array}{l}\text { Sample 1 positive } \\
\text { Sample 2 negative }\end{array}$ & $\begin{array}{l}\text { Both } \\
\text { negative }\end{array}$ \\
\hline $\begin{array}{l}\text { IgM aCL } \\
\begin{array}{l}n=17 \\
\text { IgG aCL }\end{array}\end{array}$ & 5 & 4 & 3 & 5 \\
$n=17$ & 3 & 5 & 1 & 8 \\
\hline
\end{tabular}

rone), one developed erythema multiforme (the patient without pneumonia) and one (Down syndrome) had repeated hospital admissions. No patient died. Of the patients treated at home, 7 had evidence of pneumonia and 6 had upper respiratory tract infections or influenza-like illnesses. Five patients ( 1 hospitalized, 4 managed at home) had prolonged $(>2$ months) post-infectious respiratory symptoms and malaise. No other complications were recorded. In the control infection group 9 patients were hospitalized and 12 treated at home (not significantly different from mycoplasma group $\chi^{2}=3.5, P>0.05$ ).

The distribution of positive anticardiolipin titres in the home treated and hospitalized groups were shown in Table III. Significantly more patients in the hospitalized group had IgM anticardiolipin titres $\geqslant 30$ units. The 3 patients with a complicated course had titres of IgM and IgG anticardiolipin of $47 \mathrm{U}$ and $20 \mathrm{U}$ (facial nerve palsies), 35 and 31 (Down syndrome, repeated admissions) and $33 \mathrm{U}$ and $14 \mathrm{U}$ (erythema multiforme).

Cold agglutinins were measured in 12 patients and were positive in 5 (all greater than 1:512). IgM anticardiolipin (but not IgG) titres were significantly higher in the group with positive cold agglutinins (Figure 2). There was, however, a significant positive correlation in the mycoplasma group between IgM and IgG anticardiolipin levels (Spearman's rank correlation coefficient $r_{\mathrm{s}}=0.45$ $(n=58): P<0.01)$. The hypothesis was therefore examined that high titres of IgM anticardiolipin might be due to IgM rheumatoid factor (IgM RF) binding to low titre IgG anticardiolipin. IgM RF was measured in 23 patients; the 16 patients with the highest IgM anticardiolipin titres and 7 patients with titres in the normal range. Positive results (mainly low titre) were found in 8/16 patients with high levels and $1 / 7$ with low (see Figure 3 ). There was, however, no simple relation between IgM RF and anticardiolipin titre and the three patients with the highest anticardiolipin titres had no detectable IgM RF in this assay.

The presence of other autoantibodies was also assessed in these 23 patients. Two patients had low titre $(1 / 20)$ antinuclear antibodies, with a speckled pattern (interestingly, these were the patients with
Table III Comparison of aCL titres in home vs hospitalized groups

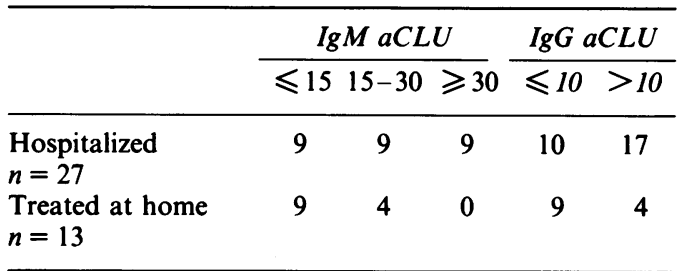

For IgM aCL, comparing patients above and below $30 \mu P=0.03$. For IgG aCL, above and below $10 \mathrm{U}$ $P=0.12$ (Fisher's exact test).

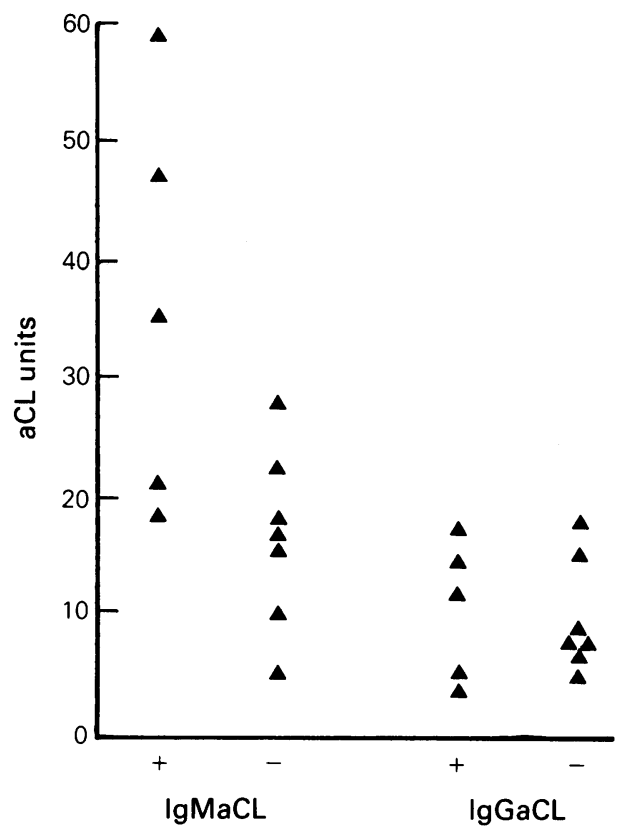

Figure 2 Levels of IgM and IgG aCL in patients with $(+)$ and without $(-)$ cold agglutinins. For IgM aCL the levels are significantly higher in the $(+)$ group $(P=0.01$ Mann Whitney U test).

the facial nerve palsies and erythema multiforme). Two patients had smooth muscle antibodies at $1 / 20$ (anticardiolipin titres IgM $59 \mathrm{U}$ and $41 \mathrm{U}$ and IgG $5 \mathrm{U}$ and $14 \mathrm{U}$ respectively). 


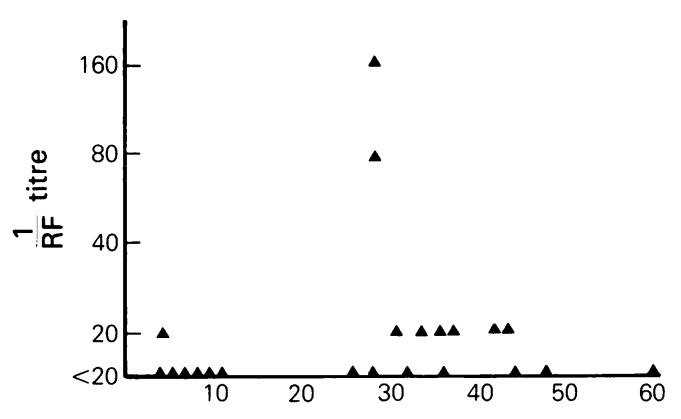

IgM aCL units

Figure 3 Relation between IgM aCL and IgM rheumatoid factor.

\section{Discussion}

The series of patients with mycoplasma infection presented here is comparable in clinical spectrum to a recently published retrospective review, ${ }^{18}$ although our rate of complications is somewhat lower (neurological problems $1 / 40$ versus $2 / 47$, erythema multiforme $1 / 40$ versus $2 / 47$. No cases of arthritis, hepatitis or pericarditis were detected). The susceptibility of patients with Down syndrome was also noted in the retrospective series.

We have confirmed the presence of antiphospholipid antibodies in acute infection. Our results suggest that these antibodies occur in more than $50 \%$ of patients with mycoplasma infection and that these patients may develop higher levels of antibody when compared to individuals with other infections (although this should be interpreted cautiously, as the range of infections in the control group was low and was limited to those infections serologically investigated in a hospital virology laboratory. There is also a suggestion, although not statistically significant, that the control group had less severe disease).

These results contrast with those of Vaarala $e t$ al. ${ }^{19}$ who found no selective increase among patients with mycoplasma infection. However, the anticardiolipin assay in that study used Tween 20 (a detergent) as a washing and blocking agent. In our experience this removes most of the phospholipid from the microtitre plates.

Examination of the IgG anticardiolipin responses particularly in the paired sera, suggest that the IgM response is associated with subsequent development of $\mathrm{IgG}$ in around $50-70 \%$ of cases. The results from the paired sera also suggest that the IgM response is sustained to at least 7-14 days in the majority of cases. We feel this validates the use of principally convalescent sera in this series.

It is unlikely that the high IgM responses seen in this study can be explained in terms of rheumatoid factor activity, although some amplifying effect of rheumatoid factor cannot be ruled out in the cases in which it was present.

Two contrasting models (not entirely mutually exclusive) can be developed for the relationship between antiphospholipid antibodies and $\boldsymbol{M}$. pneumoniae infection. Firstly, the antibodies may be representative of the low grade immune responses (principally IgM, often low affinity and highly cross-reactive) to intracellular constituents seen in tissue damage due to many causes. ${ }^{10.20}$ The specificity for mycoplasma infection, the tendency to develop IgG antibodies and the low levels of other autoantibodies would tend to argue against this. Secondly, the antibody production may reflect some particular tendency of $M$. pneumoniae to stimulate autoantibody production (whether by antigenic cross-reactivity on some other immunomodulatory effect). The correlation with cold agglutinin production (usually identified with IgM anti- $\mathrm{I}^{21}$ ) is interesting. It is perhaps worthy of note that the 4 patients in which other autoantibodies were detected by immunofluorescence were all cases with high anticardiolipin binding and/or extrapulmonary complications.

The results presented here suggest that elevations in anticardiolipin titre are more marked in patients with severe infection (assessed in terms of the need for hospital admission). There is a possible correlation of antiphospholipid titre with cold agglutinin production, but the numbers are small and warrant further study.

The possibility that high levels of anticardiolipin binding may be predictive (and even possibly pathogenetic) of extrapulmonary complications in $M$. pneumoniae infection deserves further consideration. The numbers presented here do not justify such a conclusion but it is interesting to note that the two patients with the facial nerve palsies and the stroke had the second and third highest IgM anticardiolipin titres.

It has often been speculated (but rarely substantiated, except in the case of anti- $\mathrm{I}^{21}$ ) that the extrapulmonary features of $M$. pneumoniae infection may have an autoimmune basis. In one of the few studies to address this question directly ${ }^{24}$ a patient with mycoplasma infection and transverse myelitis was found, by indirect immunofluorescence, to have antibodies directed against central nervous system neurones. The patient improved with plasma exchange. Intriguingly, this patient also had a circulating inhibitor of in vitro coagulation, with features similar to the lupus anticoagulant. As indicated above, this property is very closely linked to the possession of antiphospholipid antibodies. There are also suggestions that antiphospholipid antibodies from patients with SLE may bind neuronal membrane lipids. ${ }^{25}$ It is unfortunate that, because of the retrospective nature of 
our study, we were not able to assess our patients for lupus anticoagulant activity. In patients with SLE this seems to be a more specific predictor of vascular and other complications. ${ }^{26}$

A recent retrospective study has identified infection as an important independent risk factor in patients with ischaemic stroke aged under 50 (relative risk 14.5 in the month following infection). ${ }^{27}$ The explanation for this is not clear and although changes in haematological variables (such as fibrinogen concentration and platelet count) occurring as acute phase responses could be implicated, the role of antiphospholipid antibodies would seem to be worthy of further investigation (no cases or controls in this study, from Helsinki, ${ }^{27}$ had mycoplasma infection, suggesting the incidence was low at the time of study). It would be interesting to know whether other infections (such as the principally bacterial infection recorded in the Finnish $^{27}$ study) are capable of producing antiphospholipid responses of a degree comparable with those seen in mycoplasma infection - or whether certain individuals produce a marked increase in aPL titre regardless of the infecting organism.

To what extent are the antiphospholipid antibodies seen in $M$. pneumoniae infection comparable to those seen in SLE and the primary antiphospholipid syndrome? This question needs to be answered in both quantitative and qualitative terms. Quantitatively the positive responses in our patients fall into the 'medium positive' range defined by Harris (IgM $=10-80$ 'Manchester units' $\operatorname{IgG}=6-30$ 'Manchester units'). Values within this range are associated with thrombosis and other SLE complications, although less so than values in the 'high positive' range. ${ }^{30}$ However, our patients tended to have principally IgM anticardiolipin, whereas complications in lupus and lupus-like patients are more strongly associated with $\mathrm{IgG}^{28}$ (but have been reported in patients with only IgM anticardiolipin ${ }^{29}$ ).

Qualitatively, antiphospholipid antibodies are undoubtedly heterogeneous in terms of antigen binding. An infection strongly associated with

\section{References}

1. Asherson, R.A \& Harris, E.N. Anticardiolipin antibodies clinical associations. Postgrad Med J 1987, 62: 1081-1087.

2. Oppenheimer, S. \& Hoffbrand, B.I. Optic neuritis and myelopathy in systemic lupus erythematosus. Can J Neurol Sci 1986, 13: 129-132.

3. Harris, E.N. Gharavi, A.E., Tincani, A. et al. Affinity purified anticardiolipin and anti-DNA antibodies. J Clin Lab Immunol 1985, 17: 155-162.

4. Derksen, R.H.W.M., Beisma, D., Bauma, B.N. et al. Discordant affects of prednisone on anticardiolipin antibodies and the lupus anticoagulant. Arthritis Rheum 1986, 29: $1295-1296$ antiphospholipid antibodies is syphilis. However, the phospholipid binding found here has a different antigenic specificity ${ }^{22}$ (for neutral and positively charged lipids) from that found in SLE, and is poorly detected by solid phase anticardiolipin assays such as the ELISA described here (reference 23 and unpublished results from our laboratory on 4 high titre VDRL sera, kindly supplied by Mr D. Ellis of the Central Serology Laboratory, Withington Hospital). It therefore seems likely that the antiphospholipid antibodies in mycoplasma infection have a specificity for negatively charged lipids but insufficient data are presented here to draw further comparisons with SLE.

Finally it should be noted that there are some important clinical differences between the extrapulmonary features of mycoplasma infection and the lupus related antiphospholipid antibody syndrome. Neurological disease is relatively common and thrombotic events relatively rare in mycoplasma infection ${ }^{18}$ whereas the reverse is true in SLE and in the phospholipid antibody syndrome. ${ }^{5}$ The thrombocytopenia and fetal loss noted in SLE have not been reported in mycoplasma disease.

It would thus seem unreasonable to draw too close a parallel between these two groups of disorders, and their relation to antiphospholipid antibodies. However, it would be precipitate to consider antiphospholipid antibody responses in $M$. pneumoniae infection (and possibly other infections) as an entirely irrelevant epiphenomenon.

\section{Acknowledgement}

We wish to thank Dr W.D.W. Rees for permission to study the patient with stroke and mycoplasma infection, Dr M.R. Haeney for the use of this patient's sera, Miss J. Ditchfield for the St Thomas' reference sera, Mrs E. Crosdale for help with sample location, Dr M.E. Ellis for initially drawing our attention to these antibodies in mycoplasma infection and the many physicians in the North West who allowed us to study their patients and provided clinical details. We are also grateful to Dr S.J. Richmond for her permission to use her diagnostic index of patients.

5. Harris, E.N., Asherson, R.A. \& Hughes, G.R.V. Antiphospholipid antibodies - autoantibodies with a difference. Ann Rev Med 1986, 39: 261-271.

6. Hughes, G.R.V., Harris, E.N. \& Gharavi, A.E. The anticardiolipin syndrome. J Rheumatol 1986, 13: 486-489.

7. Misra, R., Venables, P.J.W., Watkins, R.P.F. \& Maini, R.N. Autoimmunity to cardiolipin in infectious mononucleosis. Lancet 1987, ii: 629.

8. Di Prima, M.A., Sorice, M., Vullo, V., Mastroianni, C.M., Amendolea, M.A. \& Masala, C. Anticardiolipin antibody in the acquired immunodeficiency syndrome: a marker of Pneumocystis carinii infection? J Infection 1989, 18: 100-101. 
9. Klemp, P., Cooper, R.C., Strauss, F.J., Jordaan, E.R., Przybojewski, J.Z. \& Nel, N. Anticardiolipin antibodies in ischaemic heart disease. Clin Exp Immunol 1988, 74: 254-257.

10. Manoussakis, N.M., Tzioufas, A.G., Sillis, M.P., Pange, P.J.E., Goudevenos, J. \& Mountsopoulos, H.M. High prevalence of anticardiolipin and other autoantibodies in a healthy elderly population. Clin Exp Immunol 1987, 69: 557-565.

11. Isenberg, D.A., Maddison, P., Swana, G. et al. Profile of autoantibodies in the serum of patients with tuberculosis, klebsiella and other gram-negative infections. Clin Exp Immunol 1987, 67: 516-523.

12. Carreras, L.O., Defreyn, G., Machin, S.J. et al. Arterial thrombosis, intrauterine death and the lupus anticoagulant: detection of immunoglobulin interfering with prostacylin formation. Lancet 1981, i: 244-246.

13. Twomey, J.A. \& Espir, M.L.E Neurological manifestations and Mycoplasma pneumoniae infection. Br Med J 1979, 2: $832-833$.

14. Dowd, A.B., Grace, R. \& Rees, W.D.E. Cerebral infarction associated with Mycoplasma pneumoniae infection. Lancet 1987, ii: 567

15. Mulder, L.J.M.M. \& Spriengs, E.L.H. Stroke due to DIC Mycoplasma pneumoniae infection. Lancet 1987, ii: 1152-1153.

16. Loizou, S., McRea, J.D., Rudge, A.C., Reynolds, R., Boyle, C.C. \& Harris, E.N. Measurement of anticardiolipin antibodies by ELISA: standardization and quantitation of results. Clin Exp Immunol 1985, 62: 738-745.

17. Harris, E.N., Gharavi, A.E., Patel, S.P. \& Hughes, G.R.V. Evaluation of the anticardiolipin antibody test: report on an international workshop held 4 April 1986. Clin Exp Immunol 1987, 68: 215-222.

18. Ali, N.J., Sillis, M., Andrews, B.E., Jenkins, P.F. \& Harrison, B.D.W. The clinical spectrum and diagnosis of Mycoplasma pneumoniae infection. $Q J$ Med 1986, 58: 241-251.

19. Vaarala, O., Palosuo, T., Kleemola, M. \& Aho, K. Anticardiolipin responses in acute infections. Clin Immunol Immunopathol 1986, 41: 8-15.
20. Isenberg, D. \& Shoenfeld, Y. The origin and significance of anti-DNA antibodies. Immunol Today 1987, 8: 279-282.

21. Mollison, P.L., Engelfriet, C.P. \& Contreras, M. Blood Transfusion in Clinical Medicine. Blackwell Scientific Publications, Oxford, pp. 419-421.

22. Colaco, C.B. \& Male, D.K. Antiphospholipid antibodies in syphilis and thrombotic subset of SLE: distinct profiles of epitope specificity. Clin Exp Immunol 1985, 59: 449-457.

23. Harris, E.N., Gharavi, A.E., Loizou, S. et al. Cross reactivity of antiphospholipid antibodies. J Clin Lab Immunol 1985, 16: $1-6$.

24. Cotter, F.E., Bainbridge, D. \& Newland, A.C. Neurological deficit associated with Mycoplasma pneumoniae reversed by plasma exchange. $\mathrm{Br}$ Med J 1983, 286: 22.

25. Harris, E.N., Englert, H., Derve, E. et al. Antiphospholipid antibodies in acute Guillain-Barré syndrome. Lancet 1983, ii: $11361-11362$

26. Derksen, R.H.W.M., Hasselaar, P., Blokzijl, L., Gmelig Meyling, F.H.J. \& De Groot, P.G. Coagulation screen is more specific than the anticardiolipin antibody ELISA in defining a thrombotic subset of lupus patients. Ann Rheum Dis 1988, 47: 364-377.

27. Syrjanen, J., Valtonen, V.V., Ilvanainen, M., Kaste, M. \& Huttunen, J.K. Preceding infection as an important risk factor for ischaemic brain infarction in young and middle aged patients. $\mathrm{Br} \mathrm{Med} J$ 1988, 296: $1156-1160$.

28. Gharavi, A.C., Harris, E.N., Asherson, R.A. \& Hughes, G.R.V. Anticardiolipin antibodies: isotype distribution and phospholipid specificity. Ann Rheum Dis 1987, 46: 1-6.

29. Drew, P., Asherson, R.A., Zuk, R.J., Goodwin, F.J. \& Hughes, G.R.V. Aortic occlusion in SLE associated with antiphospholipid antibodies. Ann Rheum Dis 1987, 46: 612-616.

30. McHugh, N.J., Maymo, J., Skinner, R.P., James, I. \& Maddison, P.J. Anticardiolipin antibodies, livedo reticularis and major cerebrovascular and renal disease in SLE. Ann Rheum Dis 1988, 47: 110-115. 